\title{
Production and spectroscopy in heavy flavour
}

\author{
Artem Maevskiy ${ }^{1, \star}$ \\ On behalf of the ATLAS Collaboration \\ ${ }^{1}$ Skobeltsyn Institute of Nuclear Physics (SINP MSU), 1(2), Leninskie gory, GSP-1, Moscow 119991, Rus- \\ sian Federation
}

\begin{abstract}
A wide programme of studies of heavy flavour production at the LHC is performed with the ATLAS detector. This paper covers recent results in measurements of pair $b$-quark production, di- $J / \psi$ production cross-section and effective cross-section from double parton scattering. Studies on the production of $\psi(2 \mathrm{~S})$ and $X(3872)$ are presented as well. Paper also covers the measurement of $J / \psi$-meson non-prompt production fraction and $B^{ \pm}$-meson mass reconstruction.
\end{abstract}

\section{Introduction}

Studies of heavy flavour production in $p p$-collisions at the LHC provide the means for both verifying predictions of perturbative quantum chromodynamics (pQCD) and Monte-Carlo (MC) models tuning. Understanding the mechanisms of heavy flavour production is also crucial for accurate description of background channels in Standard Model (SM) measurements and new physics searches. This paper briefly covers several results in this area obtained by the ATLAS experiment [1] in $p p$ collisions at $\sqrt{s}=8 \mathrm{TeV}$ (sections 2 to 4 ) and $\sqrt{s}=13 \mathrm{TeV}$ (sections 5 and 6).

\section{Measurement of $b$-hadron pair production}

The mass of the $b$-quark introduces an additional scale for QCD calculations, which can be introduced at different stages to the factorisation into parton distribution functions, hard matrix elements and softer parton shower components. Therefore, the optimal settings must be determined by comparisons to data. Previous measurements of $b \bar{b}$ pair production have highlighted differences between theoretical predictions, especially in the small-angle $b \bar{b}$ production region, motivating further study.

Pair production of $b$-hadrons was measured in the ATLAS experiment using a $11.4 \mathrm{fb}^{-1} \mathrm{pp}$ data sample collected at $\sqrt{s}=8 \mathrm{TeV}$ [2]. Events were selected where one $b$-hadron decayed to $J / \psi(\rightarrow \mu \mu)+X$ and the other to $\mu+Y$, resulting in three muons in the final state. To probe $b$-hadron production, several differential cross sections were measured, based on the kinematics of the $J / \psi$ and the third muon. These are:

- the azimuthal separation between the $J / \psi$ and third muon, $\Delta \phi(J / \psi, \mu)$;

- the transverse momentum of the three-muon system, $p_{\mathrm{T}}(J / \psi, \mu)$;

^e-mail: artem.maevskiy@cern.ch 
- the separation between the $J / \psi$ and the third muon in the azimuth-rapidity plane, $\Delta R(J / \psi, \mu)$;

- the separation in rapidity between the $J / \psi$ and the third muon, $\Delta y(J / \psi, \mu)$;

- the magnitude of the average rapidity of the $J / \psi$ and the third muon, $y_{\text {boost }}$;

- the mass of the three-muon system, $m(J / \psi, \mu)$;

- the ratio of the transverse momentum of the three-muon system to the invariant mass of the threemuon system, $p_{\mathrm{T}} / m$, and its inverse.

The measured cross sections as a function of $\Delta R$ and $\Delta \phi$ are shown in Fig. 1 (for other variables see [2]).

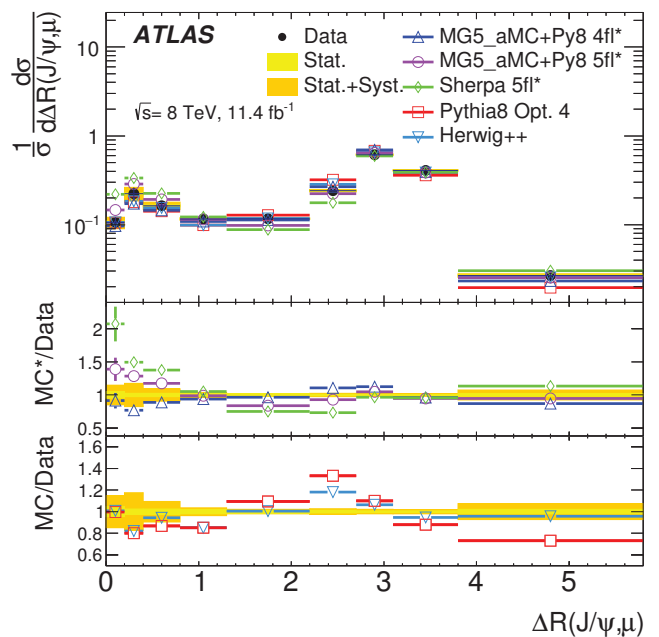

(a)

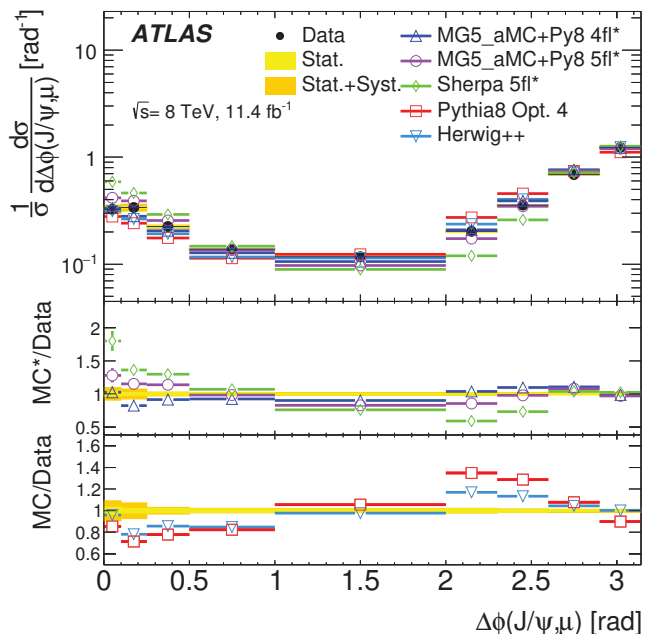

(b)

Figure 1: Measured normalised differential cross sections as a function of $\Delta R(J / \psi, \mu)$ (a) and $\Delta \phi(J / \psi, \mu)(\mathrm{b})[2]$.

ATLAS results were compared with PүтніA8 predictions, exploring several different options for the $g \rightarrow b \bar{b}$ splitting kernel, as this process dominates the region of particular interest: smallangle $b$-hadron production. The best agreement was obtained with a $p_{\mathrm{T}}$-based scale splitting kernel [2], which is shown in Fig. 1. Figure 1 also shows predictions of other generators: HeRWIG++, MadGraPh5_AMC@NLOv2.2.2 at leading order in QCD interfaced to the PyThIA8.186 parton shower model and SHERPA2.1.1. The best overall agreement is obtained with the 4-flavour MADGRAPH5_AMC@NLO+PYTHIA8, although none of the models can well-describe all the kinematic properties.

\section{Measurement of the prompt $J / \psi$ pair production}

The study of simultaneous production of two prompt $J / \psi$ mesons offers an opportunity to test our understanding of non-perturbative quantum chromodynamics. Di- $J / \psi$ events can be produced from 
a single gluon-gluon collision via a single parton scattering (SPS) or from two independent partonparton scatters in a single proton-proton collision, double parton scattering (DPS). Differential production cross section for pairs of prompt-prompt $J / \psi$-mesons as a function of various kinematic variables was measured by the ATLAS experiment using $11.4 \mathrm{fb}^{-1}$ of $\sqrt{s}=8 \mathrm{TeV}$ data collected in 2012 [3]. A data-driven method was used to extract SPS-weighted and DPS-weighted distributions of the kinematic variables, allowing the extraction of SPS and DPS components from the measured cross sections. Measured total differential cross sections along with the DPS components are shown in Fig. 2.

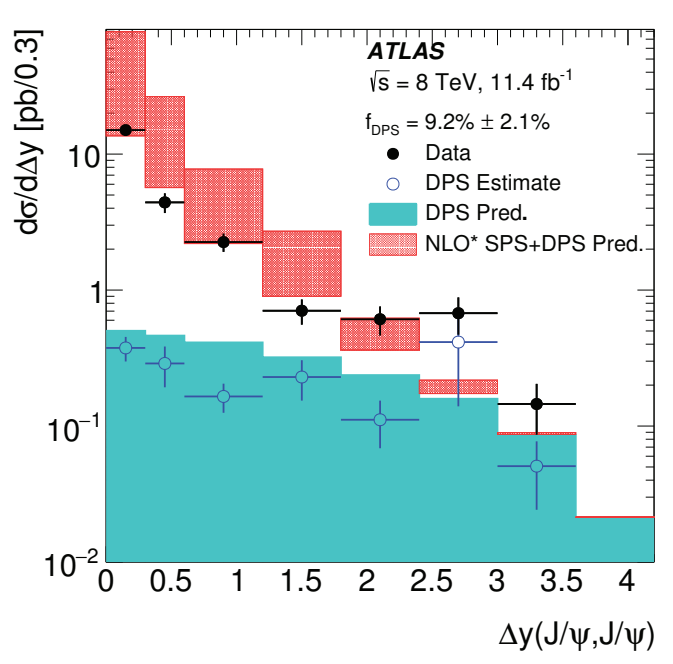

(a)

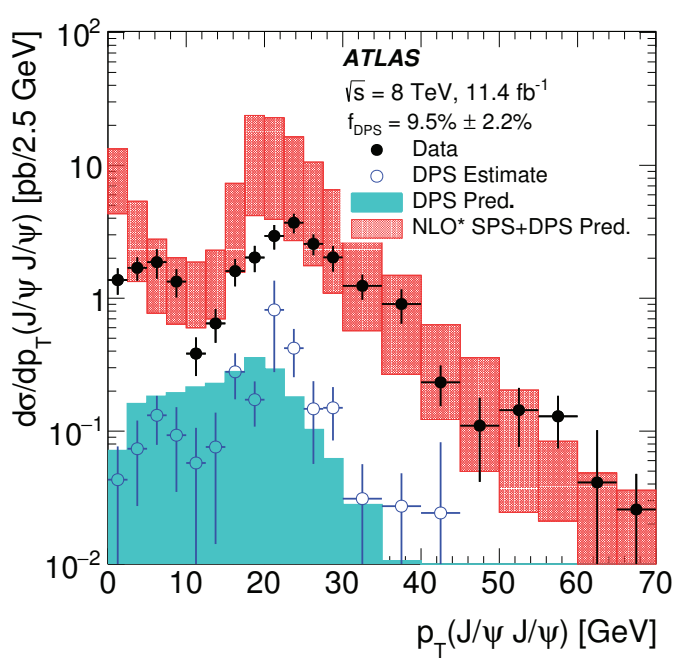

(b)

Figure 2: The DPS and total differential cross sections as a function of rapidity difference between the two $J / \psi$-mesons (a) and the transverse momentum of the di- $J / \psi$ (b) [3].

In Fig. 2, the DPS components and the total distributions are compared to LO DPS and sum of the LO DPS and NLO* SPS predictions [3]. One can see that the shape of the data-driven DPS distribution approximately agrees with the shape of DPS predictions, while there is disagreement between the total data distribution and the total theory predictions at large $\Delta y$ and low- $p_{\mathrm{T}}$ region that corresponds to di- $J / \psi$ production in an away topology.

With the measured inclusive di- $J / \psi$ cross section $\sigma_{J / \psi J / \psi}$ and the fraction of DPS events, $f_{\mathrm{DPS}}=$ $(9.2 \pm 2.1$ (stat) \pm 0.5 (syst) $) \%$, as well as the prompt $J / \psi$ cross-section, $\sigma_{J / \psi}$, the effective crosssection can be derived:

$$
\sigma_{\mathrm{eff}}=\frac{1}{2} \frac{\sigma_{J / \psi}^{2}}{\sigma_{\mathrm{DPS}}^{J / \psi, J / \psi}}=\frac{1}{2} \frac{\sigma_{J / \psi}^{2}}{f_{\mathrm{DPS}} \times \sigma_{J / \psi J / \psi}} .
$$

This value was measured to be:

$$
\sigma_{\text {eff }}=6.3 \pm 1.6(\text { stat }) \pm 1.0(\text { syst }) \pm 0.1(\mathrm{BF}) \pm 0.1 \text { (lumi) } \mathrm{mb} .
$$

This value is compared with such values obtained in various processes by various experiments in Fig. 3, as a function of centre-of-mass energy, $\sqrt{s}$. One can see that $\sigma_{\text {eff }}$ obtained in di- $J / \psi$ and 
$J / \psi \Upsilon$ measurements are lower than that obtained in other processes, which gives us a hint of possible process-dependence of DPS rates.

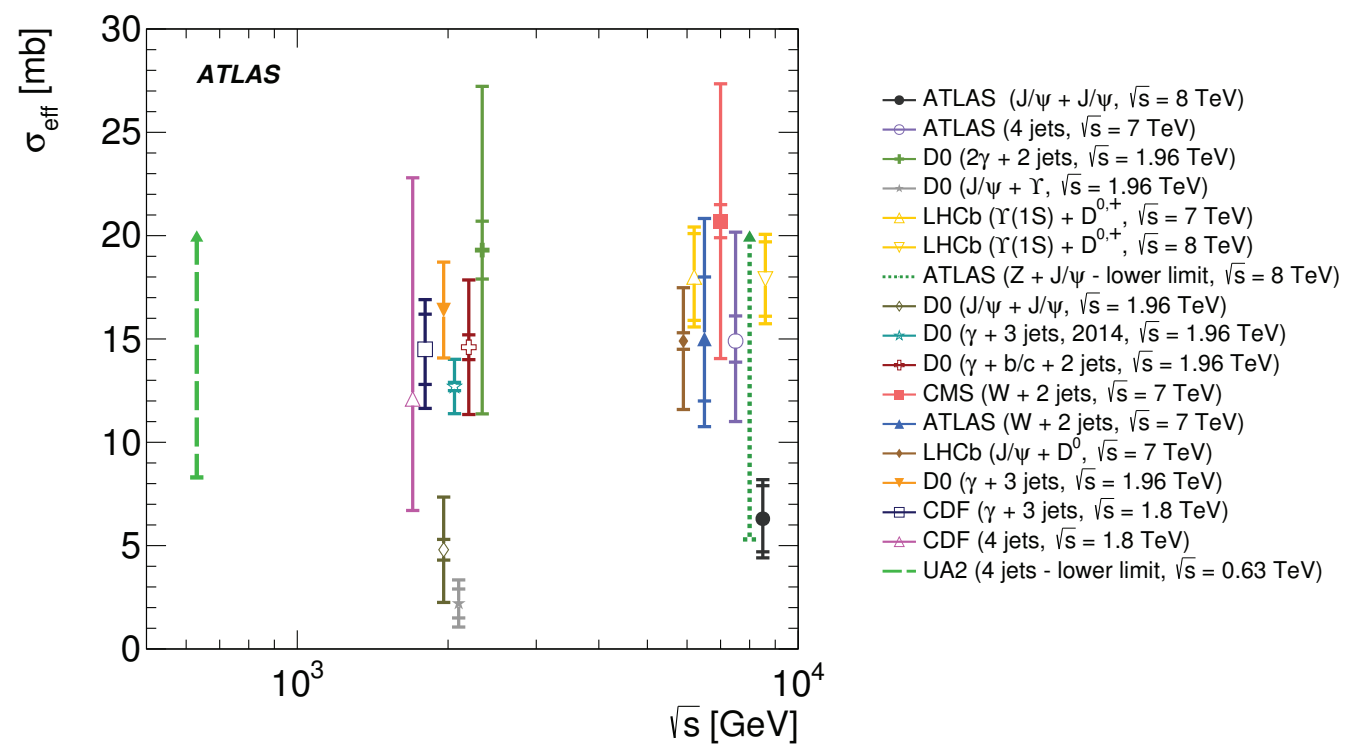

Figure 3: The effective cross section of DPS as a function of $\sqrt{s}$ [3].

\section{Production measurements of $\psi(2 S)$ and $X(3872)$}

The hidden-charm state X(3872) was discovered by the Belle Collaboration in 2003 [4]. Due to its mass being close to the $D^{0} \bar{D}^{* 0}$ threshold, it was hypothesised that this state is a $D^{0} \bar{D}^{* 0}$-molecule with a very small binding energy. However, the non-relativistic QCD (NRQCD) prediction for its production cross section, assuming the $D^{0} \bar{D}^{* 0}$-molecule hypothesis held, was too high, although the shape of the $p_{\mathrm{T}}$ dependence was described fairly well. Later, a NLO NRQCD model fitted to CMS data interpreting $X(3872)$ as a mixed $\chi_{c 1}(2 P)-D^{0} \bar{D}^{* 0}$ state, where production is dominated through the $\chi_{c 1}(2 P)$ component, showed good agreement with data.

Measuring prompt and non-prompt components of $X(3872)$ production cross section, as well as their ratios to those of $\psi(2 \mathrm{~S})$, which is fairly close in mass, may give further insight into the nature of this state and mechanisms of its production.

Differential cross sections were measured in the ATLAS experiment for the production of $\psi(2 S)$ and $X(3872)$ states in the decay channel $J / \psi \pi^{+} \pi^{-}$, using $11.4 \mathrm{fb}^{-1}$ of $p p$ collisions at $\sqrt{s}=8 \mathrm{TeV}$ [5]. The prompt and non-prompt contributions for both states are separated by analysing the displacement of the production vertex, and non-prompt production fractions are extracted, as well as $X(3872) / \psi(2 \mathrm{~S})$ production ratios separately for prompt and non-prompt components.

The measured $\psi(2 \mathrm{~S})$ cross section times branching fraction as a function of $p_{\mathrm{T}}$ of the candidate is shown in Fig. 4, where the prompt component is compared to NLO NRQCD, $k_{\mathrm{T}}$ factorisation model and the NNLO* Colour-Singlet Model (CSM) [5], while non-prompt one is compared with the predictions of FONLL calculation. One can see that NLO NRQCD and $k_{\mathrm{T}}$ factorisation models 
describe the prompt data fairly well, although there are some discrepancies at the high- $p_{\mathrm{T}}$ region. The non-prompt component is well-described by the FONLL model.
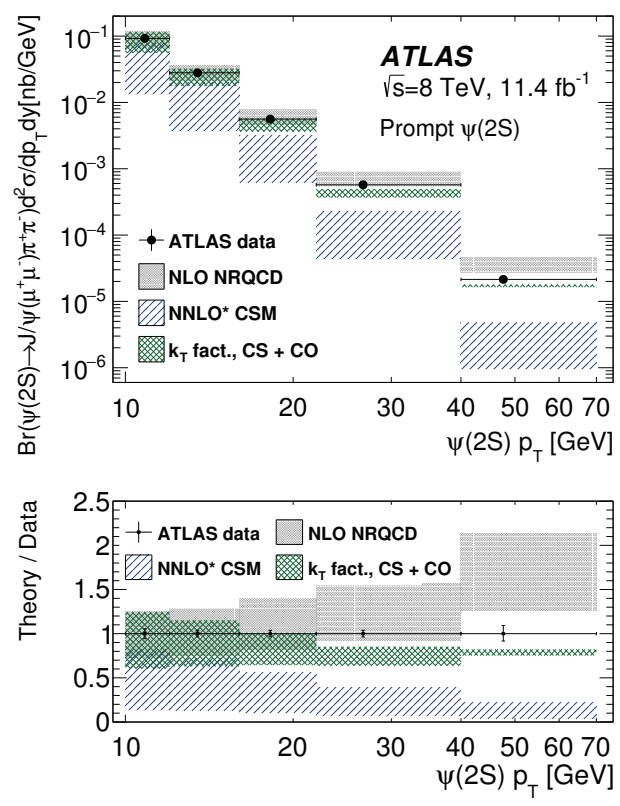

(a)
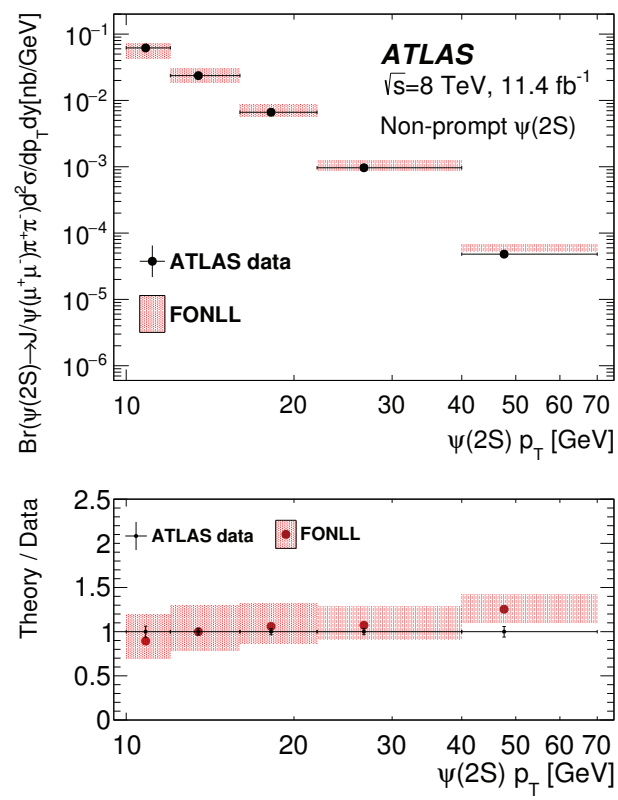

(b)

Figure 4: Measured cross section times branching fractions as a function of $p_{\mathrm{T}}$ for (a) prompt and (b) non-prompt $\psi(2 \mathrm{~S})$ production [5].

Figure 5 shows the measured cross section times branching fractions as a function of $p_{\mathrm{T}}$ for prompt and non-prompt $X(3872)$ components. The prompt component, shown in Fig. 5a, is described within the theoretical uncertainty by the prediction of the NRQCD model which, in this case, considers $X(3872)$ to be a mixture of $\chi_{c 1}(2 P)$ and $D^{0} \bar{D}^{* 0}$ molecular state, with the production being dominated by the $\chi_{c 1}(2 P)$ component and the normalisation fixed through the fit to CMS data. For the non-prompt component the FONLL model prediction for $\psi(2 S)$ was used, rescaled using simulated kinematic template for the non-prompt $X(3872) / \psi(2 \mathrm{~S})$ ratio and the effective value of the product of branching fractions $\mathscr{B}(B \rightarrow X(3872)) \mathscr{B}\left(X(3872) \rightarrow J / \psi \pi^{+} \pi^{-}\right)$estimated based on the Tevatron data (for references see [5]).

The measured non-prompt production fractions for $\psi(2 S)$ and $X(3872)$ are shown in Fig. 6, compared with CMS result. Both results agree between the two experiments within errors.

\section{Non-prompt $J / \psi$ production fraction}

The non-prompt $J / \psi$ production fraction $\sigma\left(p p \rightarrow b+X \rightarrow J / \psi+X^{\prime}\right) / \sigma\left(p p \rightarrow J / \psi+X^{\prime}\right)$ was measured in $p p$ collisions at $\sqrt{s}=13 \mathrm{TeV}$ with a data sample corresponding to an integrated luminosity of $6.4 \mathrm{pb}^{-1}$ [6]. The $J / \psi$ candidates were reconstructed in $\mu^{+} \mu^{-}$channel and their invariant mass and 

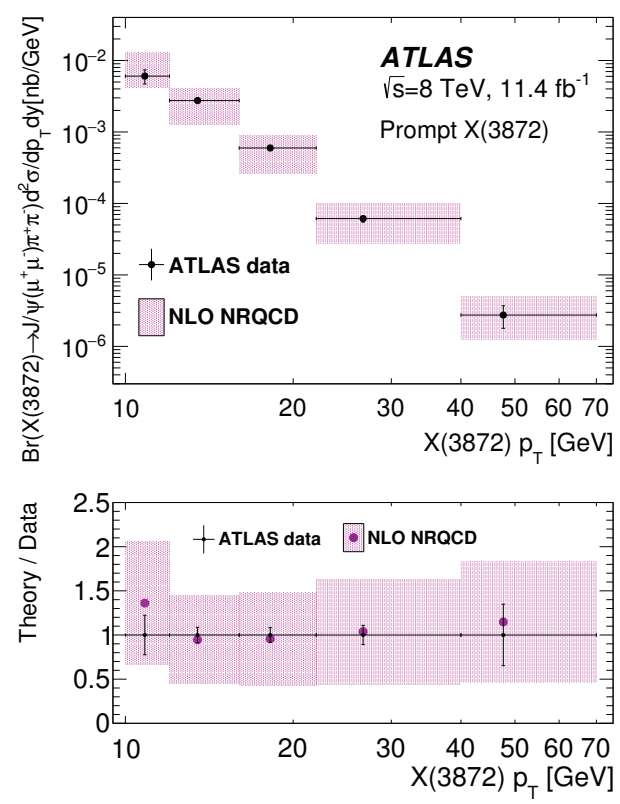

(a)
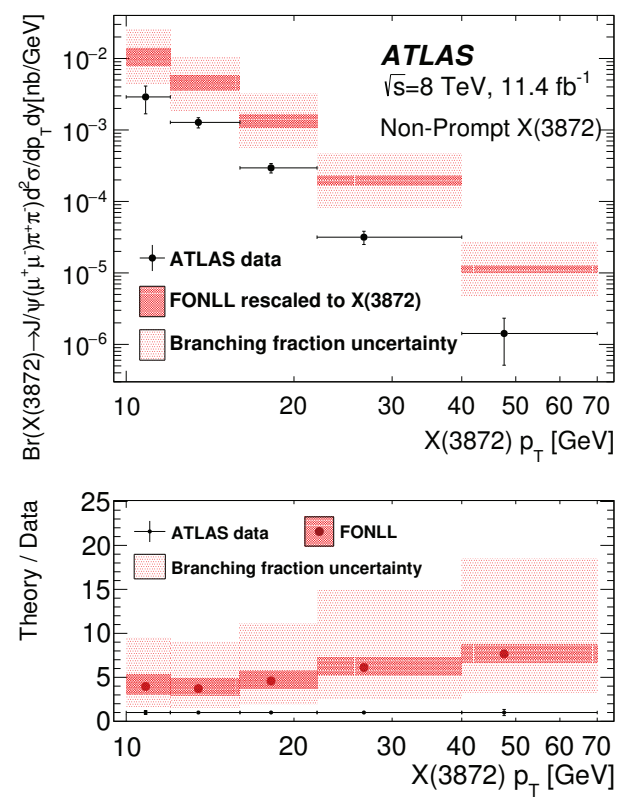

(b)

Figure 5: Measured cross section times branching fractions as a function of $p_{\mathrm{T}}$ for (a) prompt and (b) non-prompt $X(3872)$ production [5].

pseudo-proper decay time ${ }^{1}$ distributions were simultaneously fitted using an unbinned maximum likelihood fit. The extracted non-prompt fraction values are shown in Fig. 7. One can see that the fraction grows with $p_{\mathrm{T}}$ of the $J / \psi$ candidates, while no rapidity dependence can be observed at current level of precision.

\section{$6 B^{ \pm}$mass reconstruction}

Performance of the ATLAS detector in reconstructing $B^{ \pm} \rightarrow J / \psi(\mu \mu) K^{ \pm}$events was tested using $3.2 \mathrm{fb}^{-1}$ of $p p$ collision data at $\sqrt{s}=13 \mathrm{TeV}$ [7]. Events with two oppositely charged muons and one charged track were selected to form $B^{ \pm}$candidates. Their invariant mass distribution is shown in Fig. 8a. This distribution was fitted using an unbinned maximum likelihood fit with one signal and three background components. The three background components are: combinatorial, from partially reconstructed decays, and the resonant component from $B^{ \pm} \rightarrow J / \psi \pi^{ \pm}$decays. Resonant background was described by a shape derived from MC, while all other components were represented by analytical functions with free parameters. It was decided to perform fits independently in the intervals of rapidity of the $B^{ \pm}$candidates to account for varying mass resolution and to test calibration of the tracker system. The mass values extracted from these fits are shown in Fig. $8 \mathrm{~b}$ as a function of $B^{ \pm}$rapidity. One can see that deviation of each measurement from the weighted mean value of the mass is less that $0.1 \%$, which indicates good momentum calibration.

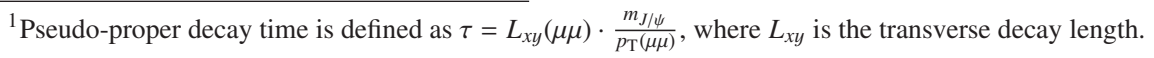




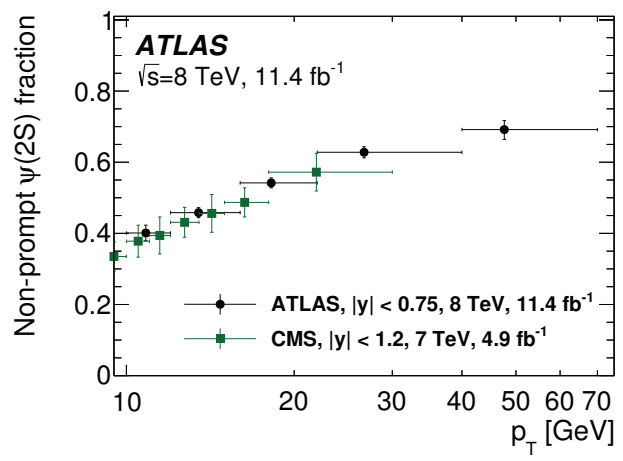

(a)

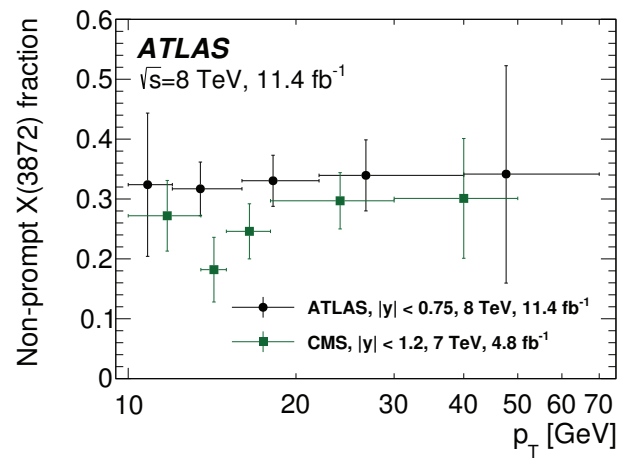

(b)

Figure 6: Measured non-prompt fractions for (a) $\psi(2 \mathrm{~S})$ and (b) X(3872) production [5], compared to CMS results at $7 \mathrm{TeV}$.

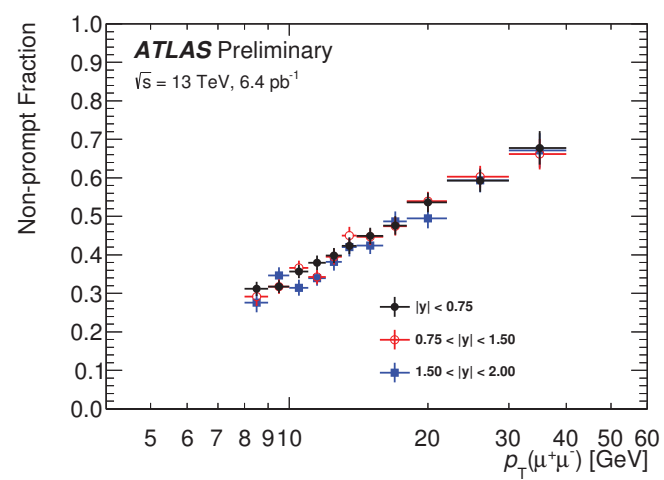

(a)

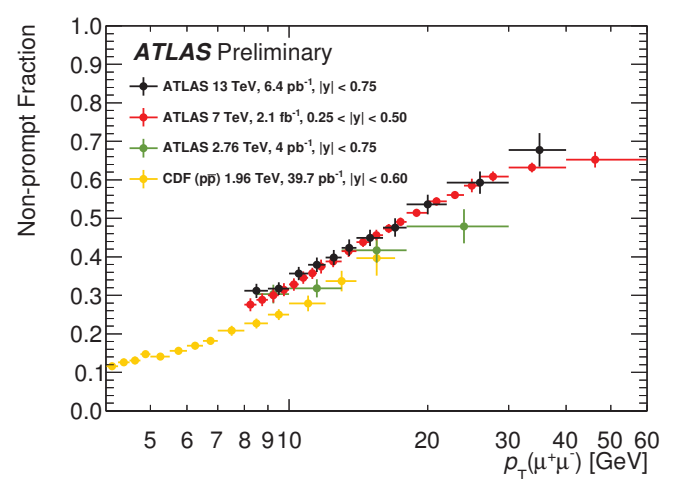

(b)

Figure 7: Measured non-prompt $J / \psi$ production fraction as a function of $p_{\mathrm{T}}(J / \psi)$ for three rapidity regions (a) and for the central rapidity region (b) compared with earlier measurements by ATLAS and $\mathrm{CDF}$ (for complete list of references see [6]).

An additional test of the fit was performed by introducing a cut on transverse decay length $\left(L_{x y}\right)$ of the candidates in order to modify the background composition. The $B^{ \pm}$mass values measured by the two versions of the fit are shown in table 1 compared with a measurement by $\mathrm{LHCb}$ [8] and with the world average [9]. One can see that all the four numbers are in good agreement. The systematic uncertainty of the ATLAS measurement is partially evaluated to be $0.25 \mathrm{MeV}$, while that value does not yet include the uncertainty related to the momentum scale of the ATLAS Inner Detector. 


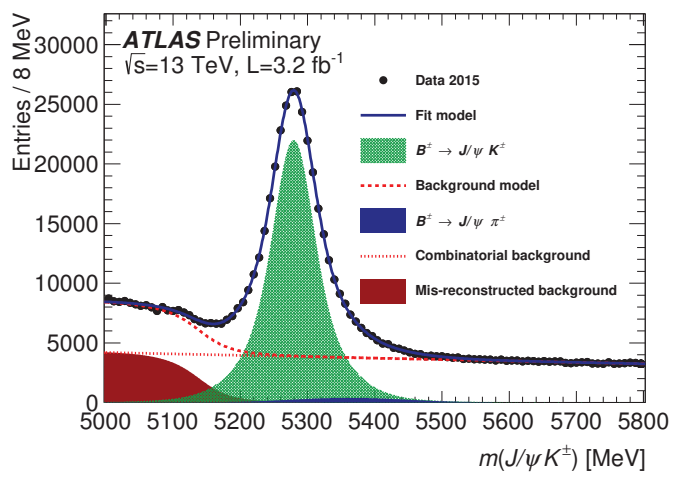

(a)

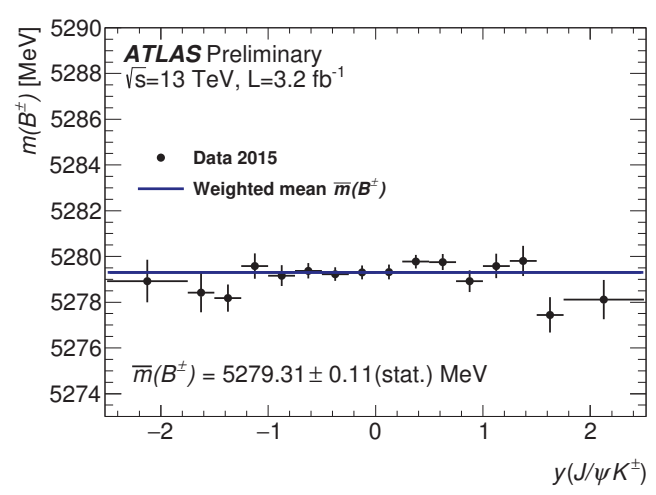

(b)

Figure 8: Invariant mass distribution of $B^{ \pm}$candidates with the maximum likelihood fit components (a) and $B^{ \pm}$mass values extracted from the fits as a function of rapidity (b) [7].

Table 1: $B^{ \pm}$mass value measured in two ways [7] compared with the world average [9] and LHCb [8].

\begin{tabular}{l|c|c}
\hline \hline Fit & $B^{ \pm}$mass $[\mathrm{MeV}]$ & Fit error $[\mathrm{MeV}]$ \\
\hline Default Fit & 5279.31 & 0.11 (stat.) \\
$L_{x y}>0.2 \mathrm{~mm}$ & 5279.34 & 0.09 (stat.) \\
\hline World Average fit & 5279.29 & 0.15 \\
LHCb & 5279.38 & 0.11 (stat.) \pm 0.33 (syst.) \\
\hline \hline
\end{tabular}

\section{Summary}

The paper covers a number of recent results in measurements of heavy flavour production. The measured pair $b$-quark differential production cross section provides new constraints to MC models parameter tuning, especially in the nearby $b$-hadron pair production region, which is of big importance for many LHC measurements. Di- $J / \psi$ production cross-section was measured at $\sqrt{s}=8 \mathrm{TeV}$ and fraction of DPS events was extracted, as well as effective cross-section from double parton scattering, which shows hints of possible process dependence of DPS. Studies on the production of $\psi(2 S)$ and $X(3872)$ were presented as well, showing good agreement between ATLAS and CMS. The nonprompt production fraction of $J / \psi$-meson and $B^{ \pm}$-meson mass were measured with $\sqrt{s}=13 \mathrm{TeV}$ data collected in 2015, showing good detector performance in new Run-2 operation conditions.

\section{Acknowledgements}

Participation in the conference was supported by the Russian Foundation for Basic Research, grants 15-02-08133 and 17-02-20235. 


\section{References}

[1] ATLAS Collaboration, JINST 3, S08003 (2008).

[2] ATLAS Collaboration, arXiv: 1705.03374 [hep-ex].

[3] ATLAS Collaboration, Eur. Phys. J. C77, 76 (2017).

[4] S.K. Choi et al. (Belle), Phys. Rev. Lett. 91, 262001 (2003).

[5] ATLAS Collaboration, JHEP 01, 117 (2017).

[6] ATLAS Collaboration (2015), ATLAS-CONF-2015-030, http://cds . cern.ch/record/2037967.

[7] ATLAS Collaboration (2015), ATLAS-CONF-2015-064, http://cds.cern.ch/record/2114830.

[8] R. Aaij et al. (LHCb), Phys. Lett. B708, 241 (2012).

[9] K. Olive et al. (Particle Data Group), Chinese Physics C 38, 090001 (2014). 